ARTICLE

Received 5 Oct 2016 | Accepted 2 Mar 2017 | Published 2 May 2017

DOI: $10.1038 /$ ncomms15127

OPEN

\title{
De novo evolved interference competition promotes the spread of biofilm defectors
}

Marivic Martin ${ }^{1, \star}$, Anna Dragoš ${ }^{1, \star}$, Theresa Hölscher ${ }^{1, \star}$, Gergely Maróti $^{2}$, Balázs Bálint ${ }^{3}$, Martin Westermann ${ }^{4}$ \& Ákos T. Kovács ${ }^{1}$

Biofilms are social entities where bacteria live in tightly packed agglomerations, surrounded by self-secreted exopolymers. Since production of exopolymers is costly and potentially exploitable by non-producers, mechanisms that prevent invasion of non-producing mutants are hypothesized. Here we study long-term dynamics and evolution in Bacillus subtilis biofilm populations consisting of wild-type (WT) matrix producers and mutant non-producers. We show that non-producers initially fail to incorporate into biofilms formed by the WT cells, resulting in 100-fold lower final frequency compared to the WT. However, this is modulated in a long-term scenario, as non-producers evolve the ability to better incorporate into biofilms, thereby slightly decreasing the productivity of the whole population. Detailed molecular analysis reveals that the unexpected shift in the initially stable biofilm is coupled with newly evolved phage-mediated interference competition. Our work therefore demonstrates how collective behaviour can be disrupted as a result of rapid adaptation through mobile genetic elements.

\footnotetext{
${ }^{1}$ Terrestrial Biofilms Group, Institute of Microbiology, Friedrich Schiller University Jena, Jena 07743, Germany. ${ }^{2}$ Institute of Biochemistry, Biological Research Centre, Hungarian Academy of Sciences, Szeged 6726, Hungary. ${ }^{3}$ Seqomics Biotechnology Ltd., Mórahalom 6782, Hungary. ${ }^{4}$ Electron Microscopy Center, Jena University Hospital, Jena 07743, Germany. ${ }^{\star}$ These authors contributed equally to this work. Correspondence and requests for materials should be addressed to Á.T.K. (email: atkovacs@dtu.dk).
} 
B iofilms, consisting of densely packed single- or multispecies communities embedded in self-produced slimy polymers, represent the most common microbial life form ${ }^{1-3}$. Several recent studies have shown that the spatial structure of biofilms has a major impact on competition and cooperation among microbes and drives evolutionary changes within microbial communities (reviewed in refs 4,5). One particularly well-studied example used static cultures of Pseudomonas fluorescens, where an oxygen gradient led to the emergence of a new wrinkly (W) phenotype that secretes polysaccharides and forms a biofilm at the air-liquid interface ${ }^{6,7}$. Interestingly, biofilms formed by $\mathrm{W}$ undergo a premature collapse caused by the incorporation of another phenotype into the biofilm without sharing the metabolic costs of exopolymer production $^{8}$. This scenario of biofilm collapse reflects the phenomenon known as 'tragedy of the commons', which happens due to invasion by non-cooperators and depletion of an overly-exploited resource (in this case the exopolymer) ${ }^{9}$.

How often the 'tragedy of the commons' happens in other biofilm communities remains an open question in sociomicrobiology. Several studies suggest that exopolymer production cannot easily be exploited by non-producing defectors ${ }^{10,11}$. Such robustness of cooperation-based biofilm formation is often explained by limited sharing of matrix components ${ }^{10-12}$, the low costs of polymer production ${ }^{11}$, the spatial assortment of cells in biofilms ${ }^{13}$ or even the intrinsic nature of certain matrix components that are exclusively shared between mother and daughter cells ${ }^{14}$. Although the key principles of certain non-producer exclusion mechanisms are becoming clear, competition experiments involving producers and non-producers are usually conducted over short timescales ${ }^{11-}$ 14 , leaving a window of opportunity for unexpected evolutionary scenarios $^{15}$. Data from various bacterial models suggest that defectors can leave a fingerprint on the evolution of social strains and promote the evolution of novel cheating-suppression mechanisms ${ }^{16}$. These can be linked to lowering the cost of cooperation by the wild-type (WT) cells ${ }^{17}$. Selection can also work to the advantage of the non-producers, which can evolve better exploitation skills ${ }^{15,17}$. In extreme cases, cooperators can be de novo selected from the population of cheats ${ }^{18}$. In general, long-term scenarios in socially heterogeneous populations of microbes are still very difficult to predict.
In this manuscript, we study the long-term social dynamics of co-cultures comprising matrix producer and non-producer strains using the widespread soil bacterium Bacillus subtilis. B. subtilis forms thick, robust structures at the air-liquid interface (pellicle) facilitated by two crucial secreted compounds: an exopolysaccharide, Eps (encoded by epsA-O), and a protein component, TasA (encoded by $\operatorname{tap} A$-sipW-tas $A$ ). In a standing culture, driven by oxygen limitation, matrix-producing strains form pellicles ${ }^{19}$. Strains lacking either one or both matrix components cannot form robust biofilms at the air-liquid interface and they barely colonize the liquid surface ${ }^{20}$. Moreover, strains producing only one of the components are able to complement each other and form a WT-like pellicle ${ }^{20}$. This strongly suggests that both matrix components secreted by producers are freely shared with non-producers and could therefore be exploited by non-producing mutants.

Here we show that on a short timescale, B. subtilis matrix non-producers have a tremendous disadvantage in co-culture with the WT. We further demonstrate how unexpected adaptive events involving mobile genetic elements can shift the social dynamics in the population and reduce biofilm formation.

\section{Results}

Biofilm non-producers are outcompeted from mixed pellicles. A positive result in a complementation assay of $B$. subtilis $\Delta e p s$ and $\Delta$ tas $A$ biofilm mutants suggests that both key biofilm components, Eps and TasA, can be shared (Fig. 1a) ${ }^{20}$. We therefore predicted that the double mutant $\Delta e p s-\Delta t a s A$, which cannot form a pellicle in monoculture ${ }^{20}$, would still be able to incorporate into the pellicle when co-cultured with the WT. To test our hypothesis, we mixed WT and $\Delta e p s-\Delta t a s A$ strains in a 1:1 ratio and allowed the pellicle to form (see Methods). The final ratio of the WT to the $\Delta e p s-\Delta \operatorname{tas} A$ strain was assessed by two alternative methods: antibiotic marker based colony forming unit (c.f.u.) counts (Fig. 1a) and fluorescence microscopy (here, GFP and mKATE2 producing WT and $\Delta e p s-\Delta \operatorname{tas} A$ mutants were used, respectively, or we used the same strains with swapped fluorescent markers; Fig. 1b,c). Surprisingly both c.f.u. assay and microscopy indicated a dramatic advantage of the WT over
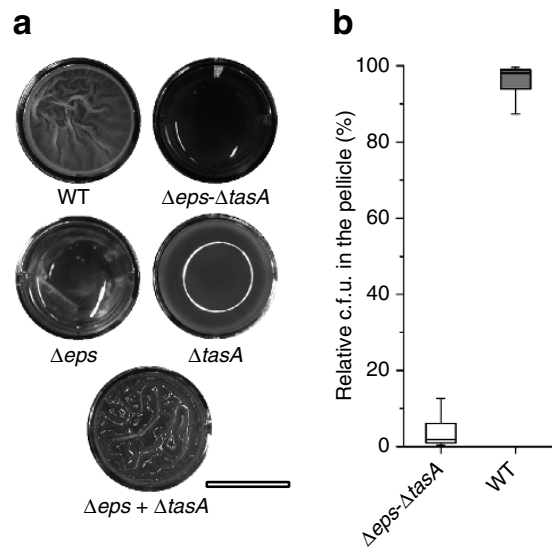

C

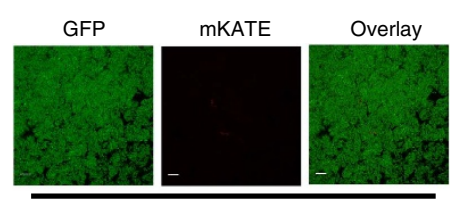

$\mathrm{WT}_{\mathrm{GFP}}$ versus $\Delta$ eps- $\Delta \operatorname{tas} A_{\mathrm{mKATE}}$

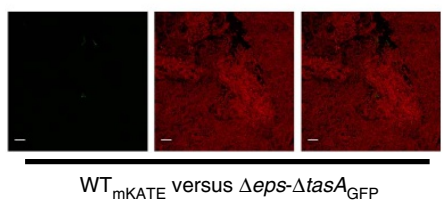

d



Figure 1 | Matrix non-producer strain does not incorporate into the wild-type pellicle. (a) Pellicle biofilms of wild-type (WT) Bacillus subtilis 168 and its mutant derivatives were recorded using an Axio Zoom microscope equipped with a black and white camera. Scale bar, $1 \mathrm{~cm}$. The reflection of the light source can be observed in the $\Delta$ tasA culture. (b) Pellicle competition assay between $\Delta$ eps- $\Delta$ tas $A$ and WT $(n=25)$. (c) Confocal microscopy images of pellicle biofilm from culture initially consisting of a 1:1 ratio of WT and $\Delta$ eps- $\Delta$ tasA (upper) and cells with swapped fluorescence protein labels (lower). Scale bars, $10 \mu \mathrm{m}$. (d) Planktonic culture competition assay between $\Delta$ eps- $\Delta$ tas $A$ and WT $(n=25)$. Boxes represent Q1-Q3, lines represent the median, and bars span from max to min. The experiments were independently repeated at least three times. ${ }^{*}$ indicates that the relative c.f.u. is significantly higher than the relative c.f.u. of $\Delta$ eps- $\Delta$ tasA ancestor in the pellicle (in panel $\mathbf{b}$ ). 
$\Delta e p s-\Delta \operatorname{tas} A$; the latter was almost completely outcompeted from the pellicle formed by the WT (Fig. 1a-c).

The incorporation success of $\Delta$ eps- $\Delta$ tas $A$ into the pellicle was positively dependent on its initial frequency (Pearson's correlation coefficient $r=0.74$; Supplementary Fig. 1A). Specifically, the mutant showed increased pellicle incorporation, up to $23 \pm 1.9 \%$, which also correlated with decline in total c.f.u. of the pellicle, but only when its initial proportion was $>50 \%$, while at initial frequencies $<50 \%$, the pellicle incorporation ranged from 0.11 to 5\% (Supplementary Fig. 1A).

To understand if the availability of nutrients influenced the ability of non-producers to incorporate into the pellicle, the competition assay was repeated using a medium in which the broth and other components were doubled $(4 \times \mathrm{SG}$, see Methods). It was observed that the $\Delta$ eps- $\Delta$ tasA strain could incorporate better in richer medium (Supplementary Fig. 1B). Using $2 \times \mathrm{SG}$, the incorporation ability of the $\Delta e p s-\Delta$ tasA strain was $2 \%$ (mean; $n=5$; s.d. $=1.37$ ), while in $4 \times \mathrm{SG}$ medium pellicle incorporation increased to $6.72 \%$ (mean; $n=5$; s.d. $=3.40$ ). Importantly, the starting ratios in these competition assays were identical $(46.72 \% \Delta e p s-\Delta t a s A ;$ mean; $n=5$; s.d. $=14.4$ ), therefore, the possibility of the initial frequency influencing these results could be excluded.

Finally, to ensure that the above result was caused by a mechanism that is specific to biofilm conditions and not simply caused by a growth defect of the $\Delta e p s-\Delta$ tasA strain, WT versus $\Delta e p s-\Delta \operatorname{tas} A$ competition experiments were also performed in planktonic cultures where oxygen distribution is more homogenous and no fitness benefits from biofilm formation are to be expected $^{11,13,21}$. In planktonic culture, $\Delta e p s-\Delta$ tas $A$ had a strong fitness advantage over the WT (Fig. 1d) that is likely due to the release of the mutant from the metabolic costs of Eps and TasA production $^{13}$, as also indicated by its higher growth rate in planktonic culture conditions (Supplementary Fig. 1C). We therefore concluded that a specific mechanism prevents incorporation of the $\Delta$ eps- $\Delta$ tasA mutant into B. subtilis pellicles.

The ratio of non-producers increases during co-evolution. For the investigation of long-term dynamics in B. subtilis WT and $\Delta e p s-\Delta$ tas A mutant co-culture over time, a serial transfer experiment was conducted in conditions promoting pellicle formation (see Methods). During the evolution experiment, two transfer methods were applied: in transfer method A, the disrupted biofilm suspension was used directly for the inoculation of fresh medium; in transfer method B the disrupted biofilm suspension was heat-treated, thereby selecting only spores for the inoculation (for detailed description see Methods). Method B was chosen to select for individuals that successfully went through the entire biofilm life cycle.

The ratio of WT to the $\Delta e p s-\Delta \operatorname{tas} A$ mutant was monitored by selective plating of frozen stocks prepared at different timepoints from the experiment, from the 2nd up to the final (10th) transfer. Despite the initial incorporation failure of $\Delta e p s-\Delta \operatorname{tas} A$ into the pellicle (Fig. 1a-c), its representation in certain populations of transfer method $\mathrm{B}$ was observed to increase dramatically over longer timescales. Remarkably, in parallel populations where transfer method B was applied (that is, selection for spores), the fraction of the $\Delta e p s-\Delta t a s A$ mutant was considerably higher after the 10th transfer than at the start of the experimental evolution in all but two replicates (Fig. 2b). Importantly, with several exceptions (in replicates 2 that remained relatively stable WT: $\Delta$ eps- $\Delta$ tasA ratio over time and replicate 4 that showed an outlying outburst of $\Delta e p s-\Delta \operatorname{tas} A$ at passage 8 ), the percentage of the $\Delta e p s-\Delta \operatorname{tas} A$ mutant was increased in each successive passage of these populations (Fig. $2 \mathrm{~b}$ ). The values rose to $>30 \%$ in general and to a maximum of around $80 \%$ after the 10th transfer of replicate 5. Also, in one out of five parallel populations that were transferred by method $\mathrm{A}$, the fraction of $\Delta e p s-\Delta \operatorname{tas} A$ was slightly higher after the 10th transfer compared to that at the beginning of the evolution experiment (Fig. 2a).

Non-producers evolve to better incorporate into the pellicle. To further investigate the evolutionary phenomena involved in improved performance of $\Delta e p s-\Delta \operatorname{tas} A$ in the evolved biofilm population, single clones of both the WT and $\Delta$ eps-4tasA mutant were isolated from three randomly chosen populations after the 10th transfer where an increase of $\Delta e p s-\Delta$ tas $A$ in the pellicle was observed (replicates 3, 4 and 5 from transfer method B) (Fig. 2b). All evolved populations and single clones that were further analysed (or genetically modified) in this study are listed in Supplementary Table 2. For clarity, we refer to evolved matrix producers (WT strains) as eMP and to the evolved matrix nonproducers $(\Delta e p s-\Delta$ tas $A)$ as eNMP.

First, to understand which of the co-cultured strains evolved to facilitate better incorporation of the mutant into the pellicle, a series of pellicle competition assays were performed. Competition assays revealed that all but one tested eNMP strains from populations $\mathrm{B} 410 \mathrm{~m}$ and $\mathrm{B} 510 \mathrm{~m}$, and one isolate from population $\mathrm{B} 310 \mathrm{~m}$, could increase their fraction within the pellicles as compared to their ancestor when co-cultured with the ancestor WT (Fig. 3a,b). This result was confirmed by both c.f.u. assay (Fig. 3a) and fluorescence microscopy (Fig. 3b). Moreover, the ancestor $\Delta e p s-\Delta$ tasA performed even worse when co-cultured with the eMP strains compared to its performance against the WT ancestor (Fig. 3). Therefore, the eMPs completely suppressed the ancestral $\Delta e p s-\Delta$ tas $A$.

The performance of three selected eMP and eNMP representatives (one from each evolved population) against the WT ancestor was additionally determined by calculating the selection rate coefficient. All eMPs showed a positive selection rate and their relative c.f.u. in the pellicle was significantly higher that 50\% (Supplementary Fig. 3). However, the ancestor $\Delta e p s-\Delta$ tas $A$ and eNMPs had negative selection rates, which indicates poor performance during competition with the ancestor WT. Nevertheless, the ancestor $\Delta e p s-\Delta$ tasA strain showed the poorest performance (selection rate value of -3.36) and all the eNMPs, $\mathrm{B} 310 \mathrm{~mA}, \mathrm{~B} 410 \mathrm{mB}$ and $\mathrm{B} 510 \mathrm{mC}$, revealed improved performance compared to the ancestor mutant strain, with selection rate values of $-2.59,-1.14$ and -2.25 , respectively.

Finally, the eNMPs were challenged with the eMPs selected from the corresponding populations (that is, $\mathrm{B} 310 \mathrm{~mA}$ versus B310wtA or B410mB versus $B 410 w t B)$. We noticed that the eNMPs from population B310m exhibited a slight decrease in pellicle incorporation compared to their pellicle incorporation when in competition with the ancestor WT (Fig. 3). Overall the eMPs performed better at suppressing the eNMPs as compared to the WT ancestor, however, certain eNMPs from populations B410m and B510m still displayed significantly improved incorporation whether competing against the evolved or ancestor WT (Fig. 3). On the basis of these competition assays, we conclude that evolutionary changes in the $\Delta$ eps- $\Delta$ tas A mutant, rather than the WT, resulted in the improved performance of the non-producers in mixed pellicles.

It was further revealed that the incorporation success of the eNMPs did not depend on their initial frequency. Competition assays with different starting ratios of the WT ancestor to each of the eNMPs $(B 310 \mathrm{~mA}, \mathrm{~B} 410 \mathrm{mB}$ and $\mathrm{B} 510 \mathrm{mC})$ revealed that the eNMPs exhibited higher levels of pellicle incorporation regardless of their starting frequency (Supplementary Fig. 1D-F). B310mA, B410mB and $\mathrm{B} 510 \mathrm{mC}$ showed an average pellicle incorporation percentage of 


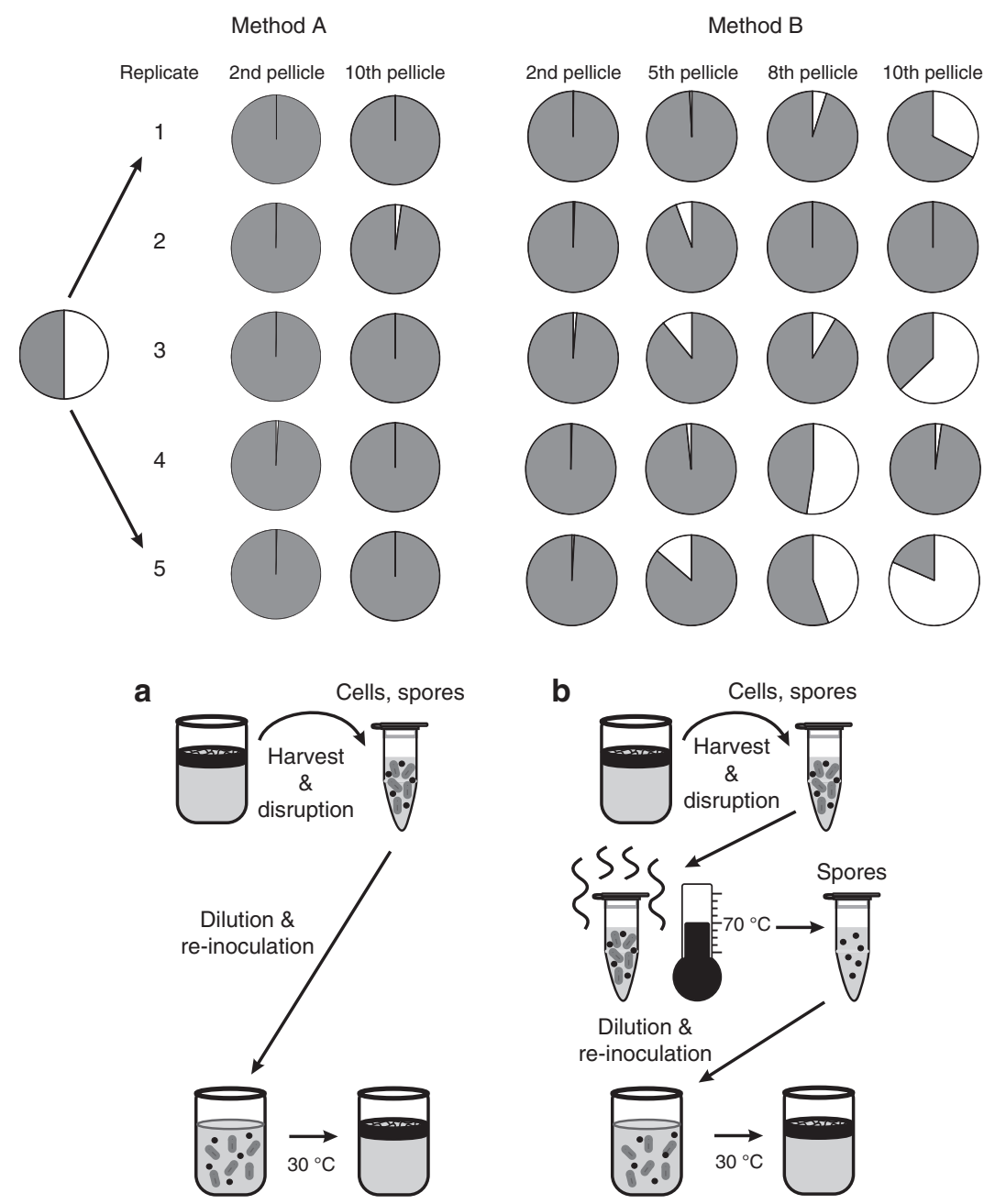

Figure 2 | Fraction of WT and $\Delta$ eps- $\Delta$ tas $\boldsymbol{A}$ strains changes during pellicle serial transfer. In transfer method (a), the pellicle was harvested, disrupted (see Methods) and the suspension of spores and cells was directly used for inoculation of new medium. After harvest and disruption, the cells in transfer method (b) were heat-treated at $70^{\circ} \mathrm{C}$ for $15 \mathrm{~min}$ to eliminate all vegetative cells. The resulting spore suspension was used to inoculate new medium and was incubated for 2-3 days to allow for pellicle formation. For transfer method (b), the gradual change of wild-type (grey) and $\Delta$ eps- $\Delta$ tas $A$ (white) ratio during the evolution experiment is also presented. The ratio was followed during evolution experiments using the disrupted pellicle suspension directly. In addition, the ratio was redetermined from frozen glycerol stocks for the second, eighth and tenth pellicles.

9.13\% (Pearson's correlation coefficient $r=0.17), 18 \%(r=0.22)$ and $27 \%(r=0.16)$, respectively (Supplementary Fig. 1D-F).

Further experiments also revealed that, in contrast to the ancestor mutant (Supplementary Fig. 1B), the incorporation percentage of the eNMP B310mA was not affected by doubling the concentration of resources in the medium (Supplementary Fig. 1B); the incorporation of the evolved B310mA was $9.54 \% \pm 3.04 \%$ in $2 \times$ SG medium and $9.88 \% \pm 2.04 \%$ in $4 \times$ SG. These results suggest that the incorporation efficiency of the eNMPs might be driven by a different mechanism from that in the ancestor $\Delta e p s-\Delta t a s A$.

Incorporation of eNMPs decreases biofilm productivity. A productivity assay was performed to understand the effect of increased incorporation of the eNMPs on the biofilm productivity and to compare the productivity of the eMPs relative to the ancestor WT. Productivity was measured by weighing the whole biomass of the pellicle and is represented as relative productivity compared with the ancestor WT (that is, ancestor $\mathrm{WT}$ productivity $=1$ ).
As expected, the productivity of the mixed pellicle consisting of the WT ancestor and mutant ancestor was very similar to the productivity of the WT ancestor grown alone, indicating that the presence of the ancestor $\Delta e p s-\Delta$ tas $A$ did not affect the biofilm productivity (Fig. 4). This result agrees with our results showing that ancestor $\Delta e p s-\Delta \operatorname{tas} A$ was almost completely outcompeted from the pellicle (Fig. 1a-c). In contrast, the productivity of pellicles containing both the ancestor WT and the eNMPs was lower than the productivity of the monoculture WT (productivity values $<1$ ), indicating that the population was negatively affected overall when eNMPs were present (Fig. 4). Interestingly, the eMPs in monocultures (B310wtA, B410wtB and B510wtC) had higher productivity than the WT ancestor (Fig. 4; Supplementary Fig. 4A). Finally, we examined the productivities of the evolved pairs with common evolutionary histories (B310wtA + B310mA; $\mathrm{B} 410 \mathrm{wtB}+\mathrm{B} 410 \mathrm{mB}$; and $\mathrm{B} 510 \mathrm{wtC}+\mathrm{B} 510 \mathrm{mC})$. For all three pairs, the productivity of the mixed pellicles was lower than the productivity of the ancestor WT; however, these differences were statistically significant only for the pairs B410wtB $+\mathrm{B} 410 \mathrm{mB}$ and B510wtC + B510mC (Fig. 4). Nevertheless, in all combinations, the eNMP + eMP productivities were significantly lower than the corresponding eMP productivity, indicating reproducible 

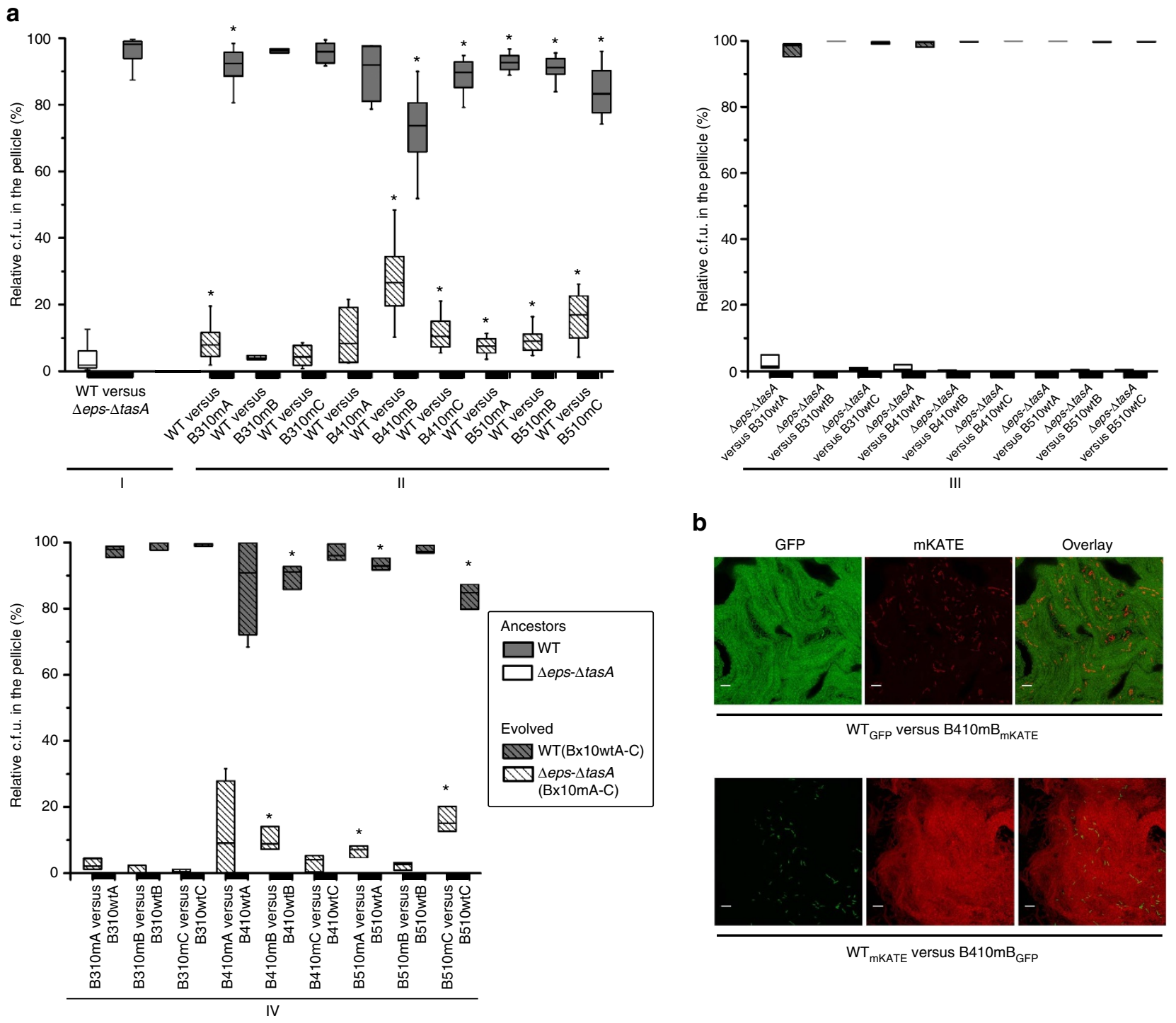

b
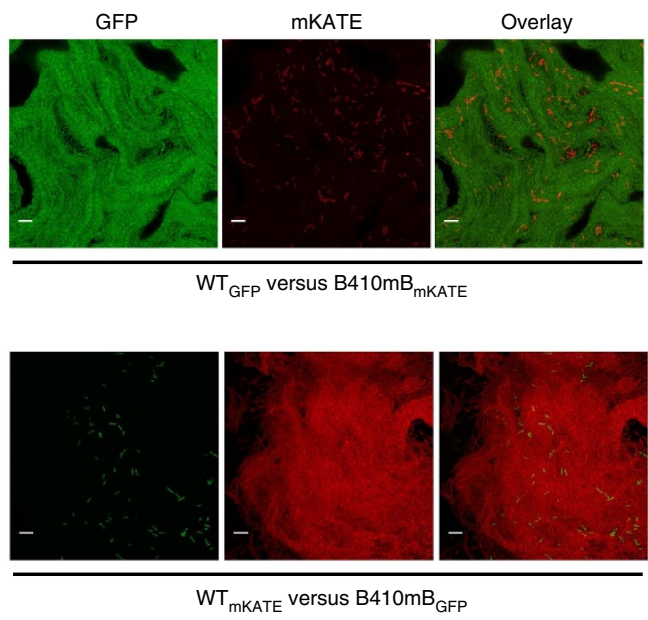

Figure 3 | Pellicle competition assay. (a) WT ancestor and $\Delta$ eps- $\Delta$ tas A mutant ancestor (I); and WT ancestor and eNMP strains 310mA, 310mB, 310mC, $410 \mathrm{~mA}, 410 \mathrm{mB}, 410 \mathrm{mC}$ and $510 \mathrm{~mA}, 510 \mathrm{mB}, 510 \mathrm{mC}$ (II). On a separate panel (above right): pellicle competition assay between WT ancestor and $\Delta$ eps- $\Delta$ tas $A$ mutant ancestor, eMP strains (three isolates per population, as in a) and $\Delta$ eps- $\Delta$ tas $A$ mutant ancestor (III). On a panel below: pellicle competition assay between WT ancestor and $\Delta$ eps- $\Delta$ tasA mutant ancestor, eMP strains and eNMP strains sharing evolutionary history (IV). Boxes represent Q1-Q3, lines represent the median, and bars span from max to min. Each competition assay was replicated in parallel with the ancestor WT versus $\Delta$ eps- $\Delta$ tas $A$ combination at least twice. ${ }^{*}$ in sections II and IV indicate that the relative c.f.u. are significantly different from the relative c.f.u. of WT versus $\Delta$ eps- $\Delta$ tasA ancestor competition. (b) Confocal microscopy images of pellicle biofilms (left) including swapped fluorescence marker proteins (right). Scale bars, $10 \mu \mathrm{m}$.

negative effects of the eNMPs on the productivity of the entire evolved population (Fig. 4). In addition, the pellicles formed by the populations from sequential evolutionary timepoints showed overall decreases in productivity in evolutionary time (Supplementary Fig. 4B), presumably caused by the increasing frequency of eNMPs in pellicles (Fig. 2b).

These results show that although matrix producers evolved a higher productivity, higher incorporation of the coevolved matrix non-producers into the pellicle eventually decreased the overall population productivity.

eMPs and eNMPs contain multiple SNPs in prophage elements. To understand the genetic basis of the observed evolutionary dynamics, the genomes of three eMP and three eNMP populations separated from the 10th transfer of method B cultures (replicates 3, 4 and 5), where the frequency of non-producers was observed to increase during evolution (either gradually or periodically), were subjected to high- throughput sequencing (Supplementary Table 2). The genomes of corresponding three single isolates of eMPs (B310wtA, B410wtB and B510wtC) and three eNMPs (B310mA, B410mB and $\mathrm{B} 510 \mathrm{mC}$ ) from those populations were also sequenced. In addition, the genomes of the WT ancestor and the $\Delta e p s-\Delta t a s A$ ancestor were resequenced to screen for any single SNPs that emerged before the evolution experiment during standard stock preparation and laboratory procedures. The sequencing of six populations (eMPs B310wt, B410wt and B510wt and eNMPs $\mathrm{B} 310 \mathrm{~m}, \mathrm{~B} 410 \mathrm{~m}$ and $\mathrm{B} 510 \mathrm{~m}$ ) and six single isolates (B310wtA, $\mathrm{B} 410 \mathrm{wtB}, \mathrm{B} 510 \mathrm{wtC}, \mathrm{B} 310 \mathrm{~mA}, \mathrm{~B} 410 \mathrm{mB}$ and $\mathrm{B} 510 \mathrm{mC})$ revealed multiple single-nucleotide polymorphisms (SNPs) exclusively accumulated in three distinct sites on the chromosome compared to the ancestors: two prophage-like regions previously described as prophage-like element 5 and prophage-like element 6 (ref. 22), and the SP $\beta$ prophage region (Supplementary Data 1; Fig. 5a,b). In population $\mathrm{B} 310$ there were $617 \mathrm{SNPs}$, while in populations B410 and B510 the number of SNPs exceeded 1000. More than 


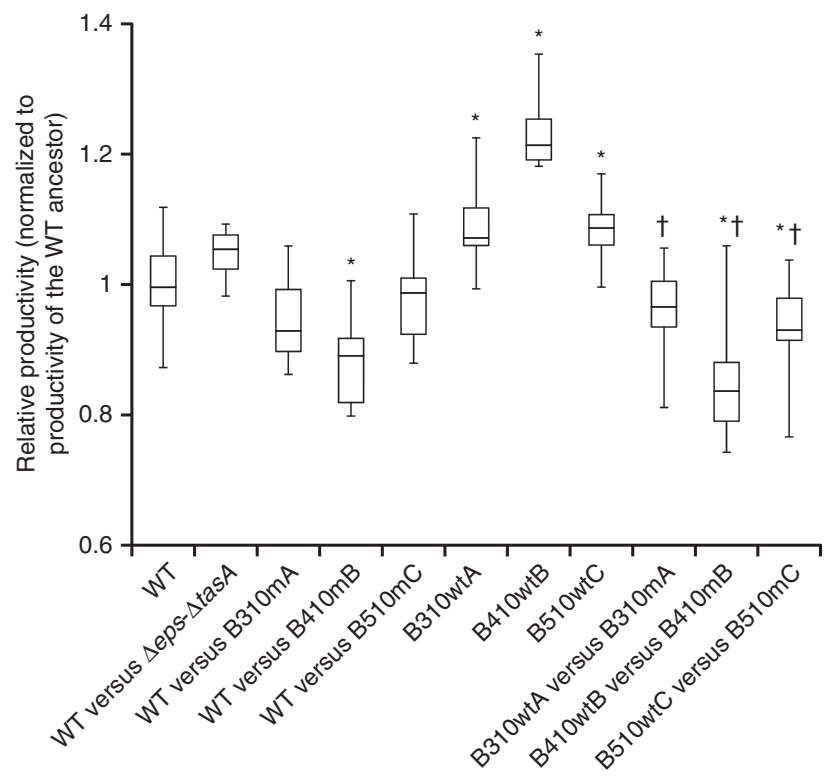

Figure 4 | Productivity assay based on biofilm biomass. Productivity of $\Delta$ eps- $\Delta$ tas A ancestor and eNMPs B310mA, B410mB and B510mC, respectively, in co-cultures with WT ancestor, monocultures of eMPs B310wtA, B410wtB and B510wtC, and co-cultures of eNMPs with corresponding eMPs compared to the WT ancestor $(n=10$; $t$-Student; two-tail $P<0.05$ ). Boxes represent $\mathrm{Q} 1-\mathrm{Q} 3$, lines represent the median, and bars span from max to min. Each WT versus co-culture/eMP comparison was replicated at least twice. ${ }^{*}$ - productivities significantly different from the WT ancestor. $\dagger$-productivities significantly lower from the corresponding eMP cultures.

$50 \%$ of SNPs detected in single isolates overlapped with SNPs found in the corresponding populations (Supplementary Data 1; Fig. 6a,b).

Further analysis of the sequenced genomes revealed that there was also a large parallel mutational overlap between the evolutionarily unrelated populations and single isolates (regardless of WT or $\Delta e p s-\Delta t a s A$ background; Supplementary Data 1; Fig. 6a,b). For visual representation of this overlap we produced a windowed-average identity score for the alignment of the entire $134 \mathrm{kbp} \mathrm{SP} \beta$ region of the evolved strains/populations to the ancestral SP $\beta$ (Supplementary Fig. 5A). A global analysis of SNPs from all six single isolates showed that the majority of SNPs represented synonymous substitutions (58\%), 17\% were non-synonymous but evolutionarily conserved (that is, similar; Blosum62 matrix score $\geq 1$ ), 9\% were evolutionarily nonconserved and non-synonymous (Blosum62 matrix score $\leq 0$ ), and the remaining $16 \%$ of the substitutions were located in noncoding regions (Supplementary Fig. 5B). We also compared the distributions of SNPs in the eMPs and eNMPs by analysing the functions of affected genes. We observed that eMPs accumulated more SNPs than the corresponding eNMPs, especially in genes related to the toxin production and secretion (Supplementary Data 1; Supplementary Fig. 5D,E). However, most of the affected genes belonged to the unknown function category.

More detailed analyses of the sequencing data on the evolved strains suggested duplications of certain genome fragments and genome rearrangements compared to the ancestors. Duplications were indicated by the increased sequencing coverage within the SNP-containing regions (Supplementary Fig. 6A) and the striking pattern of SNP frequencies (Supplementary Data 1; Supplementary Fig. 6B), which was confirmed by PCR and Sanger sequencing of the particularly highly-mutated SP $\beta$ fragment $(2,178,034-2,179,407)$ from the genomic DNA of B310mA and
B310wtA (Supplementary Fig. 6C). Interestingly, the PCR product obtained from $\mathrm{B} 310 \mathrm{~mA}$ gave a clear chromatogram with all SNPs present, whereas B310wtA showed a heterogeneous chromatogram with double peaks in the positions of SNPs, one peak coming from an ancestor-like base and the other from the evolved-like base (Supplementary Fig. 6C). In addition, the SP $\beta$ fragments could still be amplified by PCR even after deletion of the original SP $\beta$ region from the chromosomes of $\mathrm{B} 410 \mathrm{mB}$ and B510mC (Supplementary Fig. 6D-F). The identification of genome rearrangements was made after de novo assembly of sequencing reads into contigs (Fig. 6c). All of the predicted rearrangements involved sequences belonging to prophage-like elements 5 and 6 and various SP $\beta$ fragments, and included the exact regions where multiple SNPs accumulated (Fig. 6c). The presence of two randomly selected rearrangements (contig type 1 and type 4) was confirmed by PCR to occur exclusively in the evolved strains; it did not occur in the ancestor WT or ancestor $\Delta$ eps- $\Delta$ tasA (Supplementary Fig. 6G). Altogether, we conclude that the emergence of multiple SNPs in all evolved strains (both eMPs and eNMPs) was linked to duplications and rearrangements within prophage elements in the B. subtilis genome. It is important to note that the mutation frequencies of the ancestor and the evolved strains were similar, as confirmed using fluctuation assays (Supplementary Fig. 5C). The obtained mutation frequencies were comparable to previously reported data for other B. subtilis strains ${ }^{23}$, suggesting that the ancestor strains used here were not hypermutators. Moreover, when the same ancestor strain was evolved for $\sim 350$ generations in emulsion droplets, 60 SNPs and short deletions were identified (Eisha Mhatre and Á.T. Kovács, unpublished data).

Hybrid SP $\beta$ prophage shows lytic activity towards the ancestors. Rearrangements involving SP $\beta$ prophage regions have previously been described as a result of the hybridization of SP $\beta$ with another B. subtilis phage, phi3T (ref. 24). A hybrid form of SP $\beta$ can undergo spontaneous excision from the chromosome to form a pseudolysogen, or it can enter a lytic cycle leading to active phage-particle release ${ }^{24}$. To verify whether the eMPs and eNMPs in the present study spontaneously released phage particles into the medium, phages were precipitated from the supernatants of cultures of selected evolved strains and of the WT ancestor (as a negative control) and visualized by transmission electron microscopy.

No phage particles could be detected in the precipitate obtained from the WT ancestor, which was in line with previous findings ${ }^{25}$. When the WT ancestor was grown in the presence of the prophage-inducing agent mitomycin C, PBSX-like phage particles were detected in its supernatant, which again reproduced previous results ${ }^{26}$ (Supplementary Fig. 7A). However, even in the absence of mitomycin $\mathrm{C}$, the evolved strains $\mathrm{B} 410 \mathrm{mB}$ and B410wtB released two types of phage particles-PBSX-like particles with a small head and a rigid tail (assignment based on ref. 26), and SP $\beta$-like particles with a big head and a longer, flexible tail (assignment based on an image provided by Vladimir Lazarevic, Hôpitaux Universitaires de Genève, Switzerland, personal communication; Fig. 7a; Supplementary Fig. 7A). The addition of mitomycin $\mathrm{C}$ to $\mathrm{B} 410 \mathrm{mB}$ and $\mathrm{B} 410 \mathrm{wtB}$ cultures resulted in a dramatic increase in the number of SP $\beta$-like phage particles in the culture supernatants (Supplementary Fig. 7A). SP $\beta$-like particles could not be detected in the supernatant of $\mathrm{B} 310 \mathrm{~mA}^{\mathrm{SP} \beta}$ - cultures, but were still present in $\mathrm{B} 410 \mathrm{mB}^{\mathrm{SP} \beta}-$ cultures, which corresponded well with the results of molecular analysis, which indicated successful deletion of SP $\beta$ from strain $\mathrm{B} 310 \mathrm{~mA}^{\mathrm{SP} \beta}-$ but not from B410mB $\mathrm{SP} \beta$ - (Supplementary Figs $6 \mathrm{D}-\mathrm{F}$ and $7 \mathrm{~A})$. 
a

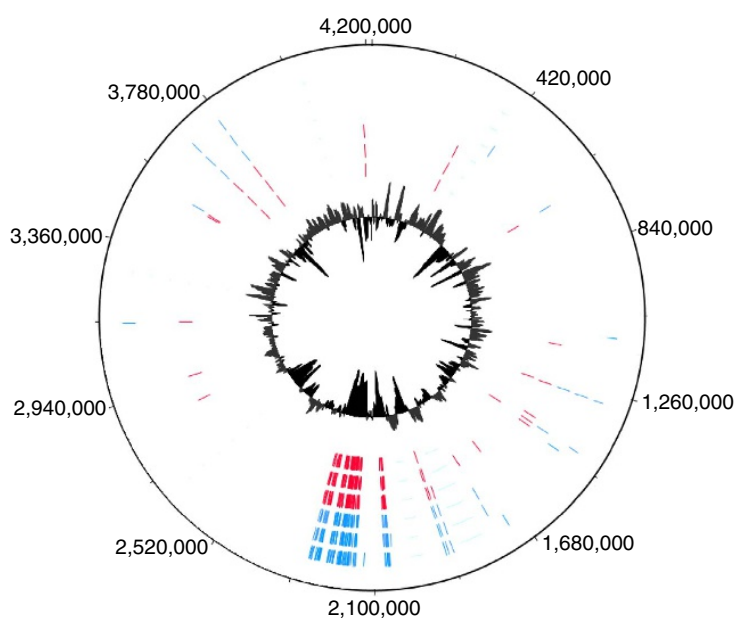

b

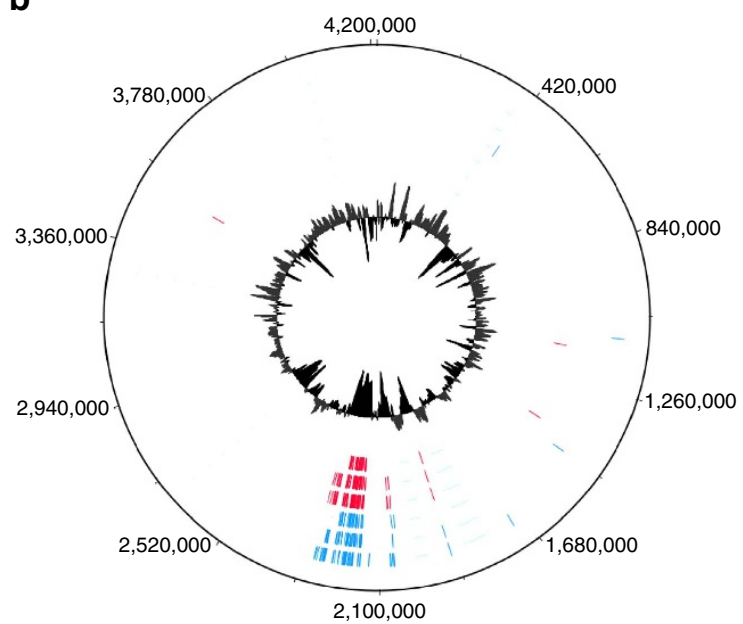

C

1.

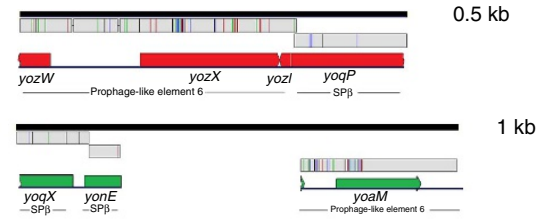

3.

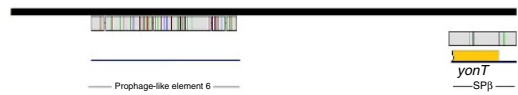

$1.2 \mathrm{~kb}$

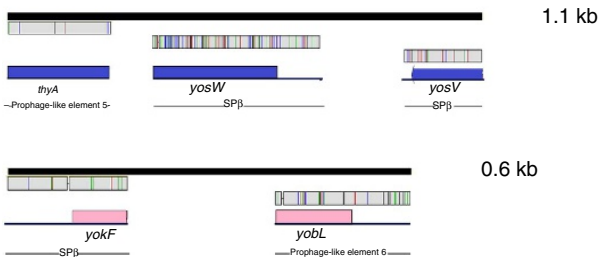

d

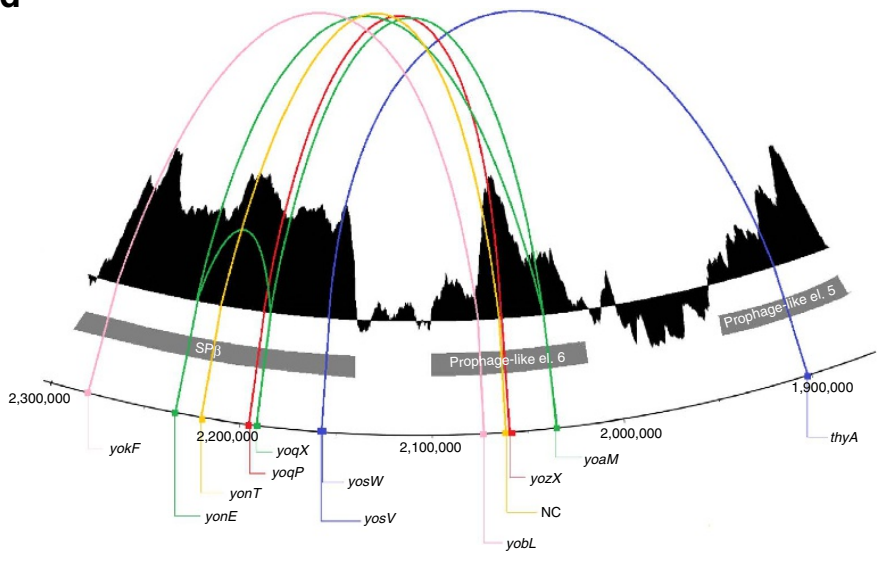

Figure 5 | Multiple SNPs and genome rearrangements are detected in the evolved strains. Genome wide distribution of SNP loci across the evolved B. subtilis populations (a) and single isolates (b) generated using the DNAPlotter tool ${ }^{56}$. (a) SNPs from six populations are presented on separate tracks; starting from the outside and moving towards the inside: B310wt, B410wt, B510wt (all shown in blue), B310m, B410m, and B510m (all shown in red). (b) SNPs in six isolates are presented on separate tracks; starting from the outside and moving towards the inside: B310wtA, B410wtB, B510wtC (all shown in blue), B310mA, B410mB, and B510mC (all shown in red). Internal black circles represent GC profiles. (c) Genome rearrangements predicted for the evolved strains based on bioinformatics analysis of the sequencing data. Black lines represent de novo assembled contigs with their sizes indicated on the right, grey boxes show aligned fragments of the reference genome (GenBank accession number AL009126), and stripes show SNP positions. Visualization was performed using Geneious software ${ }^{57}$. Corresponding genes (or non-coding regions) in the reference B. subtilis genome are shown below the alignment with different coloration used for each type of rearrangement. (d) DNA plotter graphical representation of predicted genome rearrangements (zoom view on the SP $\beta$ and prophage-like element 6 and 5 regions). The black histogram represents the GC profile, where three clusters of low-GC content directly correspond to SP $\beta$, prophage-like element 6 and prophage-like element 5. Distantly located genes that participated in rearrangements and became neighbours in the evolved strains are connected by arched lines (different colour used for each rearrangement) and are additionally marked with dots of the same colour. The coloration used for each rearrangement type corresponds to that in $\mathbf{c}$

Next, the lytic activity of the SP $\beta$ particles released by the evolved strains was tested against the ancestor strains. A series of plaque assays were performed where each strain served both as a supernatant donor and as potential prey. Neither of the ancestor strains (WT or $\Delta e p s-\Delta t a s A$ ) showed lytic activity when serving as the supernatant donor, but they were both susceptible to the lytic activity of almost all supernatants of the evolved strains (Fig. 6b, Supplementary Fig. 7B). Strain B310mA ${ }^{\mathrm{SP} \beta}-$ performed exactly the same as the ancestors, showing no lytic activity but displaying susceptibility to all supernatants, including that of B310mA (from which it was derived; Fig. 6b; Supplementary Fig. 7B). Despite the fact that all evolved strains showed lytic activity and immunity, they could be differentiated into strong (for example, B410mB) and moderate levels (for example,
B510wtC) (Fig. 6b; Supplementary Fig. 7B). The lytic activity of the supernatants of all evolved populations was assessed, including all five populations from transfer method $\mathrm{A}$ and all five populations from transfer method B (Fig. 2). Strong lytic activity towards the ancestor WT strain was found exclusively in populations that showed an increased incorporation of non-producers into the pellicle following the evolution experiment, specifically population 2 from transfer method $\mathrm{A}$, and populations 1, 3, 4 and 5 from transfer method B (Supplementary Fig. 7C). Further, populations that did not show increased incorporation of non-producers and lacked lytic activity towards the ancestor strains did not contain multiple SNPs within the SP $\beta$ regions, as confirmed by Sanger sequencing of the 2,178,034-2,179,407 genomic fragment. 
a

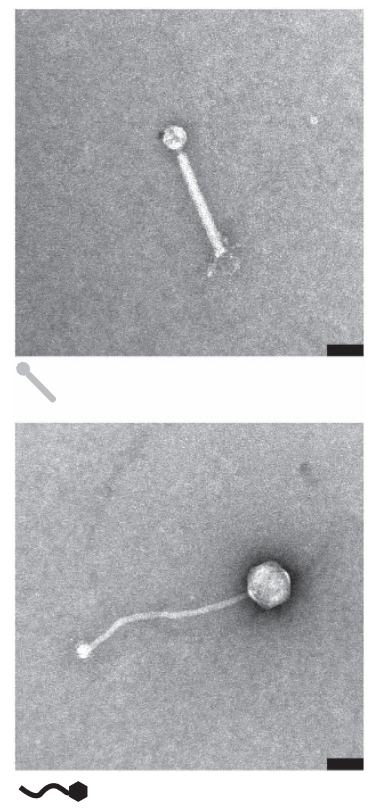

b

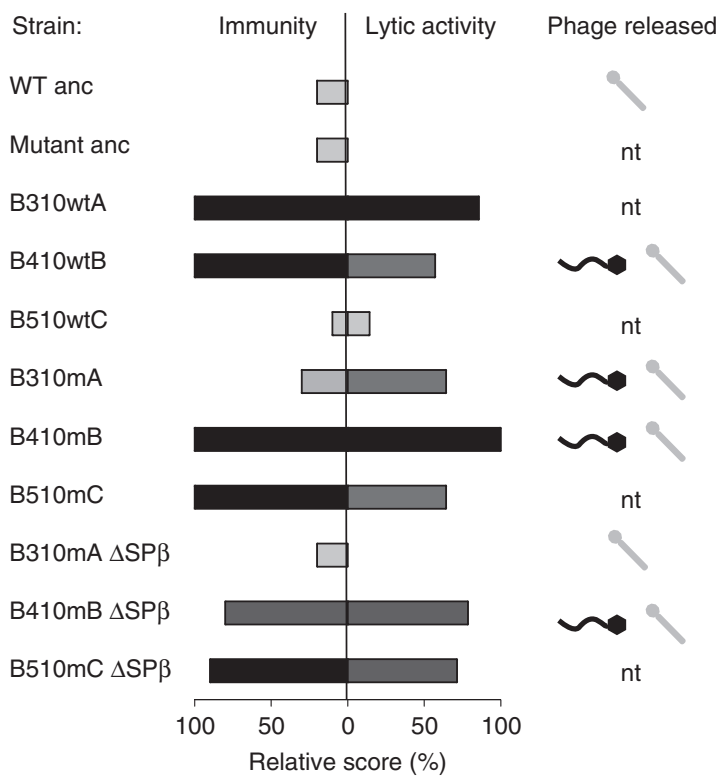

Figure 6 | Lytic phage activity appears in the evolved strains. (a) Electron micrographs of phage particles purified from B. subtilis supernatants. Upper image shows PBSX-like phage particle released by the ancestor WT only in the presence of mitomycin $C$, and by all evolved strains in the absence of mitomycin C. The lower image shows SP $\beta$-like phage particle spontaneously released by all evolved strains tested, but not by the ancestor WT or by $\mathrm{B} 310 \mathrm{~mA}^{\mathrm{SP} \beta}$ - Scale bars, $70 \mathrm{~nm}$. (b) Results of plaque assays performed with the ancestor WT, ancestor $\Delta$ eps- $\Delta$ tas $A$ and all the evolved strains, where each strain served both as a supernatant donor and as a potential host. Each strain was given 1 immunity point when resistant to a given supernatant (excluding its own supernatant), and a number of lytic activity points when showing lytic activity towards a given host (excluding itself), depending on the strength of lytic activity. Specifically, the number of points was equal to a maximal log dilution factor where lytic activity was still present (for example, a lytic activity that can still be detected in a conditioned medium diluted to $10^{-3}$ but not $10^{-4}$ is denoted with 3 points). Obtained immunity and lytic activity scores were then divided by the maximum value of each and are presented as relative percentages. Darkness of the bars is proportional to immunity/lytic activity scores values. High immunity/lytic activity scores correlated with presence of SP $\beta$-like particles (black in the right-hand column) isolated from the culture medium of those strains. 'nt' indicates media were not tested by transmission electron microscopy. The experiment was independently replicated four times.

Spontaneous phage release by the evolved strains and their lytic activity towards the ancestors suggested that higher incorporation of the eNMPs into pellicles may be the result of newly evolved interference competition. We therefore examined whether the ancestor mutant could acquire the evolved-like phenotype with higher incorporation pellicle properties through a single-phage transduction step. For the infection assay, a 1:1 mixture of the WT and $\Delta e p s-\Delta t a s A$ ancestors was introduced into standard $2 \times$ SG medium supplemented with phage precipitate obtained from $\mathrm{B} 410 \mathrm{mB}$ where the presence of SP $\beta$-like phage particles was detected. After a single growth cycle, three colonies of the WT and three colonies of $\Delta e p s-\Delta t a s A$ were isolated and their acquired lytic activity towards the ancestor strains was assessed. Finally, pellicle competition assays were performed using the WT ancestor and the three $\Delta e p s-\Delta$ tas $A$ strains isolated from the infected population (ImA, $\operatorname{ImB}$ and $\operatorname{ImC})$. As expected, the phage-infected strains behaved similarly to the evolved mutants, showing $>4$-fold (in the case of $\operatorname{ImA}$ ) and $>2.5$-fold ( $\operatorname{ImB}$ and $\operatorname{ImC})$ increased incorporation rates into the pellicle compared with the ancestor mutant (Fig. 7).

Phage release facilitates higher pellicle inclusion of eNMPs. Finally, we asked whether the presence of an identical active phage variant in both producers and non-producers is sufficient to explain the higher incorporation of the eNMPs into pellicles. This was first tested by assaying the infected mutants $(\operatorname{ImA}, \operatorname{ImB}$ and $\operatorname{ImC}$ ) with the infected WT strains (Supplementary Fig. 8). No increased pellicle incorporation of the mutants was observed, indicating that higher incorporation of the mutants cannot be explained by a general increase of phage activity in the entire population (Supplementary Fig. 8), but is due instead to subtle differences within phage elements of evolved non-producers and producers.

This was further confirmed by a fitness assay that involved $\Delta e p s-\Delta$ tasA and WT strains with an isogenic evolved background. Isogenic evolved WT and mutant strains were obtained simply by introducing the $\Delta e p s-\Delta t a s A$ deletions into eMPs. Genome resequencing confirmed that the obtained $\Delta e p s-\Delta$ tas $A$ strains still contained the genetic background of corresponding eMPs (Supplementary Data 1). When the eMPs B310wtA, $\mathrm{B} 410 \mathrm{wtB}$ and B510wtC were competed against their direct derivatives $\mathrm{B}_{10 \mathrm{wt}} \mathrm{A}^{\Delta e p s-\Delta t a s A}, \mathrm{~B}_{10} 10 \mathrm{wtB}^{\Delta e p s-\Delta t a s A}$, and $\mathrm{B} 510 \mathrm{wtC} \mathrm{C}^{\Delta e p s-\Delta t a s A}$, respectively, a very low pellicle incorporation percentage of the mutants was observed, which was comparable to the performance of the ancestor $\Delta e p s-\Delta$ tas $A$ against the ancestor WT (Supplementary Fig. 8). As expected, competition assays with the WT ancestor revealed that the transformants had comparable incorporation probabilities to the eNMPs (Fig. 7). These results indicated that although producers and nonproducers showed very similar general adaptation patterns involving major changes in mobile genetic elements, some of these changes were specific to the evolved non-producers, resulting in their improved incorporation into pellicles, most likely through an advantage in interference competition.

\section{Discussion}

Stability of cooperative interactions can determine the performance of microbes in most medically and biotechnologically relevant situations ${ }^{27-32}$. In recent years, understanding of microbial group behaviours and the mechanisms that prevent 


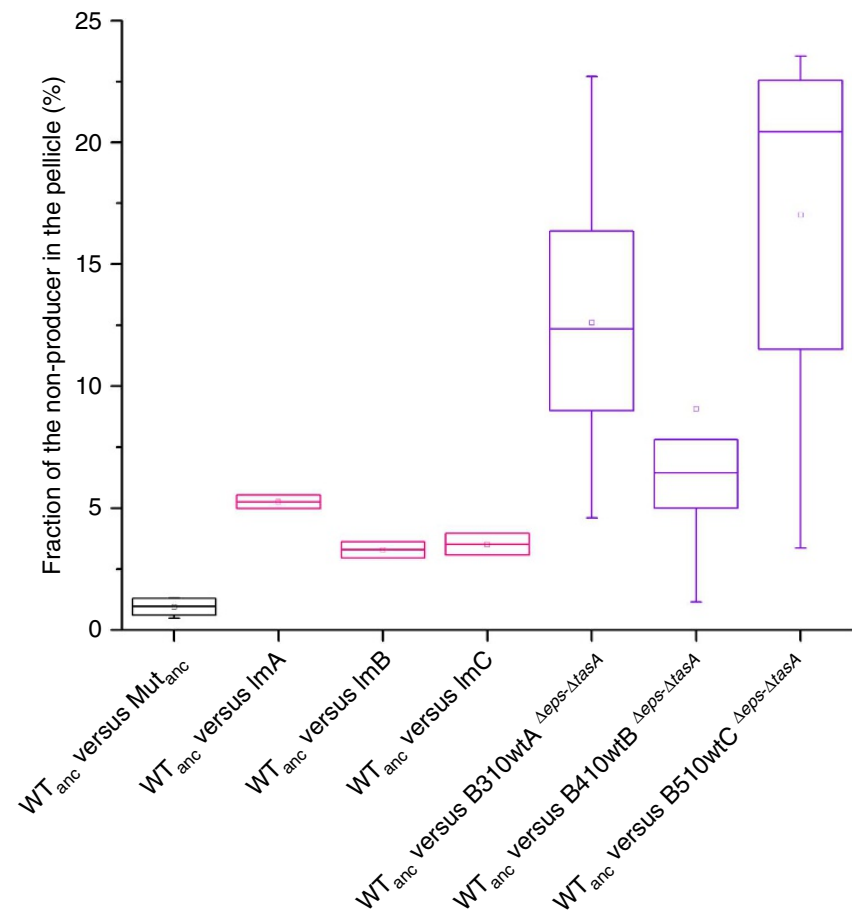

Figure 7 | Evolved mutants and ancestor mutants hosting the evolved phage have increased incorporations into the pellicle. Competition assay between the WT ancestor and $\Delta$ eps- $\Delta$ tasA ancestor (control) $(n=7)$; between the WT ancestor and three single colonies obtained after transduction of the $\Delta$ eps- $\Delta$ tas $A$ ancestor with phage particles released by eNMP B410mB $(n=2)$; and between the WT ancestor and eMPs (B310wtA, B410wtB, and B510wtC) with deleted eps and tasA ( $n=10$; $n=10 ; n=7$, respectively). Boxes represent $Q 1-Q 3$, lines represent the median, and bars span from max to min. Each competition assay in parallel with the ancestor WT versus $\Delta$ eps- $\Delta$ tas $A$ was replicated at least twice.

spreading of non-cooperative mutants has become one of the key aims of sociomicrobiology. Long timescale evolutionary experiments have already demonstrated the evolutionary plasticity of social interactions in various bacterial models $^{18,33,34}$. Here we describe a scenario where a biofilm matrix non-producer that is initially eliminated from the population increases its performance over longer timescales and evolves the ability to better incorporate into the biofilm. The evolution of improved invasion of biofilms by non-producers was previously observed by Zhang et al. ${ }^{15}$. They excluded the possibility of general adaptation being responsible for the changed social dynamics in biofilms since the evolved producer did not increase its performance towards the ancestor producer. In the present work, an increased selection coefficient and improved productivity of the evolved WT could be observed in monocultures; the same, unfortunately, could not be tested for the evolved mutants because of their inability to form pellicles in monocultures. We therefore hypothesize that the evolved increased-biofilm-incorporation-ability of the mutant was a side effect of extremely fast general adaptation of both producer and non-producers driven by mobile genetic elements. Interestingly, the improved incorporation of the non-producers into biofilms was not reproduced when both WT and non-producer strains had identical evolved genetic backgrounds (that of the evolved WT strains). This means that although the general adaptation pattern in the entire population was very similar, the nonproducers are evolutionarily ahead of the producers and carry certain specific changes that allow their improved performance in incorporation into biofilms formed by the evolved WT strains. We believe those specific differences are hidden within prophage elements of the evolved strains, and they could be revealed by de novo sequencing in the future.

In the ancestral population, the matrix non-producers ( $\Delta e p s-\Delta t a s A$, which do not secrete two key matrix components Eps and TasA) can hardly incorporate into pellicle biofilms formed by the WT. This result was rather unexpected for two reasons: first, previous work demonstrated that both Eps and TasA are shared with non-producing strains ${ }^{20}$, and, second, the production of at least one of those compounds (Eps) was proven to be costly and exploitable ${ }^{13}$. Although we did not study the competition mechanism in detail, a positive correlation between fitness, initial $\Delta e p s-\Delta \operatorname{tas} A$ frequency and resource availability suggests that in the ancestral population the growth of $\Delta e p s-\Delta \operatorname{tas} A$ is not only limited by the lack of oxygen, but also by carbon resources. We speculate that this is caused by a delay in surface co-colonization of $\Delta e p s-\Delta t a s A$, because the producer can partially privatize the matrix components. Since the WT is released from oxygen-limitation first, it can quickly deplete the remaining carbon resources, preventing further growth of the mutant. This model, however, awaits further studies.

The pellicle incorporation mechanism of evolved $\Delta e p s-\Delta t a s A$ does not depend on resource concentration or on the initial frequency of the mutant in the co-culture. It is likely that new antagonistic interactions involving infection and lysis of the ancestor WT by the evolved mutant delay surface colonization by the WT, giving the mutant a prolonged window of opportunity for co-colonization. A similar mechanism could play a role in the competition between the evolved mutant and the evolved WT, since the evolved WT strains spontaneously release phages into the medium and show a delay in pellicle formation.

How did the new lytic properties evolve? We believe that multiple rearrangements in the genomes of the evolved strains, combined with series of SNPs in regions that were rearranged, resulted in new lytic properties of the normally inactive domesticated SP $\beta$ prophage. Since this scenario was more likely to occur on sporulation treatment (that is, treatment method B), we suspect that the multiple heat-treatments involved in this treatment might have promoted phage activation ${ }^{35}$ or even rearrangements of phage elements in the genome ${ }^{36}$. The accumulation of multiple SNPs and rearrangements resembles the previously reported evolutionary response of the Streptococcus thermophilus phage to the host's CRISPR system ${ }^{37}$, however, no CRISPR/Cas has yet been identified in B. subtilis. Alternatively, rapid diversification within prophage regions combined with lytic induction may be a universal adaptive pattern of bacteria to a biofilm lifestyle, as it was previously also observed during experimental evolution of Pseudomonas aeruginosa biofilms ${ }^{38}$. Our work also demonstrates how such newly evolved phage warfare shifts social dynamics in the bacterial population in favour of biofilm non-producers. The dynamics of host-phage interactions is long studied in various experimental systems ${ }^{39}$. It was previously observed that lytic phages can shift the balance in competitive interactions by reducing the frequency of a winning partner ${ }^{40}$, or impair biofilm formation ability as a tradeoff for phage immunity ${ }^{41}$. We hypothesize that in the case of the $B$. subtilis pellicles, the disadvantage of matrix producers could originate from the degeneration of toxin/secretion-related genes in the evolved wild-types that in turn became less efficient competitors than the evolved mutants.

The improved fitness of the evolved WT strain in monoculture could be a direct result of the evolved spontaneous phage release. Normally, the excision of the SP $\beta$ prophage from the B. subtilis chromosome takes place before sporulation and allows reconstitution of the spsM gene involved in spore polysaccharide 
biosynthesis $^{42}$. Sanchez-Vizuete et al. ${ }^{43}$ demonstrated that removal of SP $\beta$ from the chromosome permanently restores $s p s M$, resulting in increased biofilm thickness. We presume that frequent spontaneous excision of SP $\beta$, or even pseudolysogeny (as demonstrated in ref. 24) in the evolved WT strains, could positively contribute to the biofilm productivity through spsM reconstitution. Excision of prophage from the host chromosome was recently linked to improved biofilm formation by Shewanella oneidensis facing cold stress ${ }^{44}$. Such a phage excision benefited the host through gene inactivation rather than reconstitution (as observed in ref. 43). Similar genetic switches triggered by prophage excision were also described in several other species (reviewed in ref. 45).

The B. subtilis SP $\beta$ prophage carries a bacteriocin-immunity system $^{46}$, several putative toxin-antitoxin systems ${ }^{47}$ and cell wall hydrolases $^{48}$. Several SP $\beta$ segments of $>250$ nucleotides exhibit $>90 \%$ identity with $B$. subtilis chromosomal regions ${ }^{25}$ promoting recombination events, especially in naturally competent strains. Not surprisingly, recent reports strongly indicate a key role of phage elements in rapid evolution of kin recognition mechanisms and antagonistic interactions between closely related, sympatric B. subtilis strains ${ }^{49,50}$. Accumulation of SNPs in the SP $\beta$ region was also observed in the evolution experiments of Overkamp et al. ${ }^{51}$, where $B$. subtilis was kept in zero-growth conditions for 42 days. Among hundreds of SNPs discovered by Overkamp et al. ${ }^{51}, 80 \%$ overlapped with the SNPs reported in this study. In addition, most of the SNPs detected were synonymous and evolutionarily conserved, suggesting selection against loss of function. Recent reports show that even non-synonymous mutations can positively contribute to fitness ${ }^{52,53}$. This again suggests that mutations or rearrangements within phage elements can be a very important evolutionary force in $B$. subtilis, with a major impact on social interactions. Recently, the profound impact of prophages on the evolution of a pathogenic bacterium was experimentally demonstrated in $P$. aeruginosa biofilms ${ }^{54}$, where the presence of phages resulted in strong selection against phage recognition elements (type IV pilus), at the same time enhancing parallel evolution ${ }^{54}$. Similar selective pressure could emerge after fast evolution of active SP $\beta$ variants in $B$. subtilis biofilms, resulting in striking parallelism in evolved populations of both WT and $\Delta e p s-\Delta \operatorname{tas} A$ bacteria.

Our work demonstrates how social dynamics in an initially very robust biofilm can be shifted by unexpected evolutionary events. We show that an adaptive genotype that is quickly tailored by mobile genetic elements can easily spread through horizontal gene transfer. The same adaptive path, although beneficial for the producer, became maladaptive in a mixed population where producers coexisted with non-producers.

\section{Methods}

Strains and cultivation conditions. Supplementary Table 1 describes strains used in this study and construction of their mutant derivatives. Strain B. subtilis 168

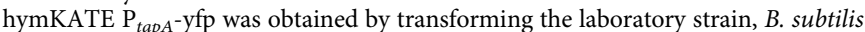
168 , with genomic DNA from DL821 and selecting for MLS resistance. Subsequently, the created strain was transformed with genomic DNA from 168 hymKate and selecting on chloramphenicol resistance and for the loss of amylase activity. The $\Delta e p s$ and $\Delta$ tas $A$ strains were obtained by transforming the 168 strains with genomic DNA isolated from DL1032 and specifically selecting for tetracycline or kanamycin resistance, respectively. The double mutant $\Delta e p s-\Delta \operatorname{tas} A$ was obtained by transforming $168 \Delta e p s$ with genomic DNA from DL1032 and selecting on the kanamycin marker. The $\Delta e p s-\Delta t a s A$ hyGFP and $\Delta e p s-\Delta$ tas $A$ hymKate strains were obtained by transforming the $\Delta e p s-\Delta$ tas $A$ strain with genomic DNA obtained from 168 hyGFP and 168 hymKate, respectively. Deletion of epsA-O and tasA genes were confirmed with PCR using oligos described in Supplementary Table 3. Strains were maintained in LB medium (Lennox broth; Carl Roth, Germany), while $2 \times$ SG medium was used for biofilm induction ${ }^{55}$.

Experimental evolution and competition assays. Experimental evolution was performed using co-cultures of fluorescently labelled but otherwise WT and
$\Delta e p s-\Delta$ tas $A$ strains grown in $2 \mathrm{ml} 2 \times \mathrm{SG}$ medium statically in a 24 -well plate at $30{ }^{\circ} \mathrm{C}$ for 2-3 days. For transfer method $\mathrm{A}$, the mature pellicles were harvested, mildly disrupted, and reinoculated after $100 \times$ dilution. For transfer method B, the pellicles were additionally heat-treated after disruption and diluted $\times 20$ during reinoculation. The sporulation frequency in the conditions applied in the evolution experiment was about $20 \%$ (Supplementary Fig. 2). To maintain similar selection bottlenecks in the two transfer methods, a fivefold lower dilution factor was used in transfer method B.

After the 2nd, 8 th and 10th pellicle transfers, frozen stocks were preserved. Evolved populations or single isolates were isolated by selecting with appropriate antibiotics. Competition experiments were performed by mixing certain ratios of 100 -fold diluted LB-pregrown cultures which were then incubated in static pellicle forming conditions for 3 days or in agitated planktonic cultures for $16 \mathrm{~h}$. The numbers of c.f.u. of the inocula and the final cultures were determined on LB-agar plates containing selective antibiotics, incubated overnight at $37^{\circ} \mathrm{C}$. Prior the c.f.u. assays, pellicles were sonicated according to a protocol optimized in our laboratory ( 2 cycles each containing $12 \times 1 \mathrm{~s}$ pulses at $20 \%$ amplitude with $1 \mathrm{~s}$ pause between the pulses), that ensured efficient disruption of biofilm clumps (as verified by microscopy) and therefore accurate total cell counts in the pellicles.

Microscopy. Bright field images of whole pellicles were obtained with an Axio Zoom V16 stereomicroscope (Carl Zeiss, Jena, Germany) equipped with a Zeiss CL 9000 LED light source and an AxioCam MRm monochrome camera (Carl Zeiss). The pellicles were also analysed using a confocal laser scanning microscope (LSM 780 equipped with an argon laser, Carl Zeiss) and Plan-Apochromat/1.4 Oil DIC M27 $\times 63$ objective. Fluorescent reporter excitation was performed with the argon laser at $488 \mathrm{~nm}$ and the emitted fluorescence was recorded at $484-536 \mathrm{~nm}$ and $567-654 \mathrm{~nm}$ for GFP and mKate, respectively. To generate pellicle images, Z-stack series with $1 \mu \mathrm{m}$ steps were acquired. Zen 2012 Software (Carl Zeiss) was used for both stereomicroscopy and CLSM image visualization.

Productivity assay. For productivity assays, pellicles were inoculated into $4 \mathrm{ml}$ of $2 \times$ SG medium placed in $35 \mathrm{~mm}$-diameter Petri dishes and incubated for 3 days at $30^{\circ} \mathrm{C}$. Next, the medium fraction was removed, and pellicles were dried at $55^{\circ} \mathrm{C}$ for $3 \mathrm{~h}$. The dry biomass was determined on an analytical balance.

Fluctuation assay. To determine the mutation rate, single colonies were picked from LB-agar medium and cultivated for $18 \mathrm{~h}$ in LB broth at $37^{\circ} \mathrm{C}$. After 100 -times dilution in $2 \times$ SG medium, cultures ( $n=10$ for each strain) were subsequently cultivated for $18 \mathrm{~h}$ with vigorous shaking, and dilution series were plated on LB-agar medium to assay the frequency of streptomycin $\left(50 \mu \mathrm{g} \mathrm{ml}^{-1}\right)$ resistant c.f.u. after $18-24 \mathrm{~h}$ at $37^{\circ} \mathrm{C}$.

Genome resequencing and genome analysis. Genomic DNA of selected populations or isolated strains was isolated using the EURex Bacterial and Yeast Genomic DNA Kit from cultures grown for $18 \mathrm{~h}$. For the evolved population, single-end fragment reads were sequenced using a Life Technologies SOLiD 5500xl sequencer. Base-calling was carried out with the software provided by the supplier. All other downstream analysis steps were done in CLC Genomics Workbench Tool 7.0.4. Reads were length-filtered, keeping only $\geq 50$ nucleotide long fragments. Mapping used only those reads that displayed $\geq 80 \%$ similarity to the reference genome (GenBank accession number AL009126) over $\geq 60 \%$ of the read length (meaning an alignment of $\geq 30$ nucleotides having $\geq 24$ identical matches). Non-specific reads were randomly placed to one of their possible genomic locations. Quality-based SNP and small in/del variant calling was carried out requiring $\geq 10 \times$ read coverage with $\geq 20 \%$ variant frequency. Only variants suggested by good quality bases $(\mathrm{Q} \geq 20)$ were taken into account. Furthermore, mutations had to be supported by evidence from both DNA strands.

For single isolate strains, paired-end fragment reads $(2 \times 250$ nucleotides $)$ were generated using an Illumina MiSeq sequencer. Primary data analysis (base-calling) was carried out with MiSeq Reporter software (Illumina). All further analysis steps were done in CLC Genomics Workbench Tool 8.0.2. Reads were quality-trimmed using an error probability of 0.05 (Q13) as the threshold. Reads that displayed $\geq 80 \%$ similarity to the reference genome (GenBank accession number AL009126) over $\geq 80 \%$ of their read lengths were used in mapping. Non-specific reads were randomly placed to one of their possible genomic locations. Quality-based SNP and small In/Del variant calling was carried out requiring $\geq 40 \times$ read coverage with $\geq 20 \%$ variant frequency. Only variants supported by good quality bases $(\mathrm{Q} \geq 20)$ were taken into account and only if they were supported by evidence from both DNA strands. Selected genomic regions were validated by Sanger sequencing (GATC Biotech, Konstanz, Germany) using oligos listed in Supplementary Table 3.

Transmission electron microscopy analysis. Selected bacterial strains were grown overnight in $\mathrm{LB}$ medium at $37^{\circ} \mathrm{C}$ with shaking at 200 r.p.m. In the case of mitomycin-C-treated cultures, mitomycin $\mathrm{C}$ was added in late exponential phase to a final concentration of $0.5 \mu^{-1} \mathrm{ml}^{-1}$. Culture supernatants were collected, mixed at a 1:4 ratio with PEG-8000 solution (PEG-8000 20\%, $2 \mathrm{M} \mathrm{NaCl}$ ), incubated on ice for at least $90 \mathrm{~min}$ and finally centrifuged $(20 \mathrm{~min}, 7,600$ r.p.m.) to obtain 
precipitate. The pellet was resuspended in $10 \%$ of the original supernatant volume in TBS solution ( $50 \mathrm{mM}$ Tris- $\mathrm{HCl}, 150 \mathrm{mM} \mathrm{NaCl}, \mathrm{pH}$ 7), incubated on ice for $90 \mathrm{~min}$ and centrifuged ( $20 \mathrm{~min}, 7600$ r.p.m.). Supernatant was carefully transferred to clean Eppendorf tubes. Purified samples $(100 \mu \mathrm{l})$ were adsorbed onto duplicate 400 mesh carbon-coated $\mathrm{Cu}$ grids (Quantifoil, Großlöbichau, Germany) for 2 min. Before use, the carbon grids were hydrophilized by $30 \mathrm{~s}$ of electric glow discharging. The grids were washed twice in distilled water and stained for $30 \mathrm{~s}$ with $1 \%$ uranyl acetate. Virus morphologies were examined using a Zeiss CEM 902A transmission electron microscope (Carl Zeiss AG, Oberkochen, Germany). At least 20 images were taken per sample at different magnifications using a $1 \mathrm{k}$ FastScan CCD-Camera (camera and software from TVIPS, Munich, Germany).

Statistical analyses. Statistical differences between two experimental groups were identified using two-tailed Student's $t$-tests assuming equal variance. Variances in the two main types of datasets (c.f.u. counts in competition assays and weight of biomass) were similar across different samples. One data point with a value greater than the mean plus 3 times the s.d. was removed from the dataset of $n>10$ as an outlier. Normal distributions within the two main data types (biomass and c.f.u.) were confirmed by Kolmogorov-Smirnov $(P>0.05)$. No statistical methods were used to predetermine sample size and the experiments were not randomized.

Data availability. The genome resequencing data are available in Supplementary Data 1. The authors declare that all other relevant data supporting the findings of the study are available within the article and its Supplementary Information files, or from the corresponding author upon request.

\section{References}

1. Costerton, J. W., Lewandowski, Z., Caldwell, D. E., Korber, D. R. \& Lappin-Scott, H. M. Microbial biofilms. Annu. Rev. Microbiol. 49, 711-745 (1995).

2. Hall-Stoodley, L., Costerton, J. W. \& Stoodley, P. Bacterial biofilms: from the natural environment to infectious diseases. Nat. Rev. Microbiol. 2, 95-108 (2004).

3. Røder, H. L., Sørensen, S. J. \& Burmølle, M. Studying bacterial multispecies biofilms: where to start? Trends Microbiol. 24, 503-513 (2016).

4. Martin, M., Hölscher, T., Dragos, A., Cooper, V. S. \& Kovács, Á. T. Laboratory evolution of microbial interactions in bacterial biofilms. J. Bacteriol. 198, 2564-2571 (2016).

5. Steenackers, H. P., Parijs, I., Foster, K. R. \& Vanderleyden, J. Experimental evolution in biofilm populations. FEMS Microbiol. Rev. 40, 373-397 (2016).

6. Rainey, P. B. \& Travisano, M. Adaptive radiation in a heterogeneous environment. Nature 394, 69-72 (1998)

7. Udall, Y. C., Deeni, Y., Hapca, S. M., Raikes, D. \& Spiers, A. J. The evolution of biofilm-forming Wrinkly Spreaders in static microcosms and drip-fed columns selects for subtle differences in wrinkleality and fitness. FEMS. Microbiol. Ecol. 91, fiv057 (2015).

8. Hammerschmidt, K., Rose, C. J., Kerr, B. \& Rainey, P. B. Life cycles, fitness decoupling and the evolution of multicellularity. Nature 515, 75-79 (2014).

9. Hardin, G. The tragedy of the commons. The population problem has no technical solution; it requires a fundamental extension in morality. Science 162, 1243-1248 (1968)

10. Xavier, J. B. \& Foster, K. R. Cooperation and conflict in microbial biofilms. Proc. Natl Acad. Sci. USA 104, 876-881 (2007).

11. Irie, Y. et al. The Pseudomonas aeruginosa PSL polysaccharide is a social but non-cheatable trait in biofilms. Preprint at bioRxiv http://dx.doi.org/10.1101/ 049783 (2016).

12. Srinandan, C. S., Elango, M., Gnanadhas, D. P. \& Chakravortty, D. Infiltration of matrix-non-producers weakens the Salmonella biofilm and impairs its antimicrobial tolerance and pathogenicity. Front. Microbiol. 6, 1468 (2015).

13. van Gestel, J., Weissing, F. J., Kuipers, O. P. \& Kovács, Á. T. Density of founder cells affects spatial pattern formation and cooperation in Bacillus subtilis biofilms. ISME J. 8, 2069-2079 (2014).

14. Nadell, C. D., Drescher, K., Wingreen, N. S. \& Bassler, B. L. Extracellular matrix structure governs invasion resistance in bacterial biofilms. ISME J. 9, 1700-1709 (2015).

15. Zhang, Q. G., Buckling, A., Ellis, R. J. \& Godfray, H. C. Coevolution between cooperators and cheats in a microbial system. Evolution 63, 2248-2256 (2009).

16. Manhes, P. \& Velicer, G. J. Experimental evolution of selfish policing in social bacteria. Proc. Natl Acad. Sci. USA 108, 8357-8362 (2011).

17. Kümmerli, R. et al. Co-evolutionary dynamics between public good producers and cheats in the bacterium Pseudomonas aeruginosa. J. Evol. Biol. 28, 2264-2274 (2015).

18. Fiegna, F., Yu, Y. T., Kadam, S. V. \& Velicer, G. J. Evolution of an obligate social cheater to a superior cooperator. Nature 441, 310-314 (2006).

19. Hölscher, T. et al. Motility, chemotaxis and aerotaxis contribute to competitiveness during bacterial pellicle biofilm development. J. Mol. Biol. 427, 3695-3708 (2015)
20. Branda, S. S., Chu, F., Kearns, D. B., Losick, R. \& Kolter, R. A major protein component of the Bacillus subtilis biofilm matrix. Mol. Microbiol. 59, 1229-1238 (2006).

21. Madsen, J. S. et al. Facultative control of matrix production optimizes competitive fitness in Pseudomonas aeruginosa PA14 biofilm models. Appl. Environ. Microbiol. 81, 8414-8426 (2015).

22. Kunst, F. et al. The complete genome sequence of the gram-positive bacterium Bacillus subtilis. Nature 390, 249-256 (1997).

23. Viret, J. F. \& Alonso, J. C. A new mutator strain of Bacillus subtilis. Mol. Gen. Genet. 208, 353-356 (1987).

24. Spancake, G. A., Hemphill, H. E. \& Fink, P. S. Genome organization of SPbeta c2 bacteriophage carrying the thyP3 gene. J. Bacteriol. 157, 428-434 (1984).

25. Lazarevic, V. et al. Nucleotide sequence of the Bacillus subtilis temperate bacteriophage SP $\beta c 2$. Microbiology 145, 1055-1067 (1999).

26. Okamoto, K. et al. Properties of the defective phage of Bacillus subtilis. J. Mol. Biol. 34, 413-428 (1968).

27. Inglis, R. F., Gardner, A., Cornelis, P. \& Buckling, A. Spite and virulence in the bacterium Pseudomonas aeruginosa. Proc. Natl Acad. Sci. USA 106, 5703-5707 (2009).

28. Itoh, T. et al. Cooperative degradation of chitin by extracellular and cell surface-expressed chitinases from Paenibacillus sp. strain FPU-7. Appl. Environ. Microbiol. 79, 7482-7490 (2013).

29. Katsuyama, C. et al. Complementary cooperation between two syntrophic bacteria in pesticide degradation. J. Theor. Biol. 256, 644-654 (2009).

30. Li, Y. H. \& Tian, X. Quorum sensing and bacterial social interactions in biofilms. Sensors (Basel) 12, 2519-2538 (2012).

31. Pollitt, E. J., West, S. A., Crusz, S. A., Burton-Chellew, M. N. \& Diggle, S. P. Cooperation, quorum sensing, and evolution of virulence in Staphylococcus aureus. Infect. Immun. 82, 1045-1051 (2014).

32. Raymond, B., West, S. A., Griffin, A. S. \& Bonsall, M. B. The dynamics of cooperative bacterial virulence in the field. Science 337, 85-88 (2012).

33. Andersen, S. B., Marvig, R. L., Molin, S., Krogh Johansen, H. \& Griffin, A. S. Long-term social dynamics drive loss of function in pathogenic bacteria. Proc. Natl Acad. Sci. USA 112, 10756-10761 (2015).

34. Velicer, G. J., Kroos, L. \& Lenski, R. E. Loss of social behaviors by Myxococcus xanthus during evolution in an unstructured habitat. Proc. Natl Acad. Sci. USA 95, 12376-12380 (1998).

35. Siegel, E. C. \& Marmur, J. Temperature-sensitive induction of bacteriophage in Bacillus subtilis 168. J. Virol. 4, 610-618 (1969).

36. Kawamura, F., Saito, H. \& Ikeda, Y. A method for construction of specialized transducing phage rho 11 of Bacillus subtilis. Gene 5, 87-91 (1979).

37. Paez-Espino, D. et al. CRISPR immunity drives rapid phage genome evolution in Streptococcus thermophilus. MBio 6, e00262-15 (2015).

38. McElroy, K. E. et al. Strain-specific parallel evolution drives short-term diversification during Pseudomonas aeruginosa biofilm formation. Proc. Natl Acad. Sci. USA 111, E1419-E1427 (2014).

39. Lenski, R. E. \& Levin, B. R. Constraints on the coevolution of bacteria and virulent phage: a model, some experiments, and predictions for natural communities. Am. Nat. 125, 585-602 (1985).

40. Brockhurst, M. A., Fenton, A., Roulston, B. \& Rainey, P. B. The impact of phages on interspecific competition in experimental populations of bacteria. BMC. Ecol. 6, 19 (2006).

41. Brockhurst, M. A., Buckling, A. \& Rainey, P. B. The effect of a bacteriophage on diversification of the opportunistic bacterial pathogen, Pseudomonas aeruginosa. Proc. Biol. Sci. 272, 1385-1391 (2005).

42. Abe, K. et al. Developmentally-regulated excision of the SPbeta prophage reconstitutes a gene required for spore envelope maturation in Bacillus subtilis. PLoS Genet. 10, e1004636 (2014).

43. Sanchez-Vizuete, P. et al. Identification of $y p q P$ as a new Bacillus subtilis biofilm determinant that mediates the protection of Staphylococcus aureus against antimicrobial agents in mixed-species communities. Appl. Environ. Microbiol. 81, 109-118 (2015).

44. Zeng, G. et al. Cold adaptation regulated by cryptic prophage excision in Shewanella oneidensis. ISME J. 10, 2787-2800 (2016)

45. Feiner, R. et al. A new perspective on lysogeny: prophages as active regulatory switches of bacteria. Nat. Rev. Microbiol. 13, 641-650 (2015).

46. Dubois, J. Y. et al. Immunity to the bacteriocin sublancin 168 Is determined by the SunI (YolF) protein of Bacillus subtilis. Antimicrob. Agents Chemother. 53, 651-661 (2009).

47. Jahn, N., Preis, H., Wiedemann, C. \& Brantl, S. BsrG/SR4 from Bacillus subtilis - the first temperature-dependent type I toxin-antitoxin system. Mol. Microbiol. 83, 579-598 (2012)

48. Sudiarta, I. P., Fukushima, T. \& Sekiguchi, J. Bacillus subtilis CwlP of the SP prophage has two novel peptidoglycan hydrolase domains, muramidase and cross-linkage digesting D-endopeptidase. J. Biol. Chem. 285, 41232-41243 (2010).

49. Lyons, N. A., Kraigher, B., Stefanic, P., Mandic-Mulec, I. \& Kolter, R. A combinatorial Kin discrimination system in Bacillus subtilis. Curr. Biol. 26, 733-742 (2016). 
50. Stefanic, P., Kraigher, B., Lyons, N. A., Kolter, R. \& Mandic-Mulec, I. Kin discrimination between sympatric Bacillus subtilis isolates. Proc. Natl Acad. Sci. USA 112, 14042-14047 (2015).

51. Overkamp, W. et al. Physiological and cell morphology adaptation of Bacillus subtilis at near-zero specific growth rates: a transcriptome analysis. Environ. Microbiol. 17, 346-363 (2015).

52. Agashe, D. et al. Large-effect beneficial synonymous mutations mediate rapid and parallel adaptation in a bacterium. Mol. Biol. Evol. 33, 1542-1553 (2016).

53. Bailey, S. F., Hinz, A. \& Kassen, R. Adaptive synonymous mutations in an experimentally evolved Pseudomonas fluorescens population. Nat. Commun. 5, 4076 (2014)

54. Davies, E. V. et al. Temperate phages both mediate and drive adaptive evolution in pathogen biofilms. Proc. Natl Acad. Sci. USA 113, 8266-8271 (2016).

55. Kobayashi, K. Bacillus subtilis pellicle formation proceeds through genetically defined morphological changes. J. Bacteriol. 189, 4920-4931 (2007).

56. Carver, T., Thomson, N., Bleasby, A., Berriman, M. \& Parkhill, J. DNAPlotter: circular and linear interactive genome visualization. Bioinformatics 25, 119-120 (2009).

57. Kearse, M. et al. Geneious Basic: an integrated and extendable desktop software platform for the organization and analysis of sequence data. Bioinformatics 28, 1647-1649 (2012).

\section{Acknowledgements}

We are grateful to Christian Kost and Michael Brockhurst for helpful discussions. B. subtilis strain SPmini was kindly provided by Prof Tsutomu Sato (Research Center of Micro-Nano Technology, Hosei University). This work was funded by grant KO4741/2-1 from the Deutsche Forschungsgemeinschaft (DFG) within the priority program SPP1617 and a Startup Fund from Jena School for Microbial Communications (JSMC). MG was supported by GINOP-2.3.2-15-2016-00011 (EU Structural Funds). T.H. and A.D. were supported by International Max Planck Research School and Alexander von Humboldt foundation fellowships, respectively.

\section{Author contributions}

A.T.K. conceived the project; M.M., A.D., T.H., and A.T.K. designed the research; M.M., A.D. and T.H. performed the research; M.M., A.D. and T.H. analysed the data; M.W. performed the electron microscopy; G.M. and B.B. performed and analysed genome resequencing; and M.M., A.D., T.H., and A.T.K. wrote the paper.

\section{Additional information}

Supplementary Information accompanies this paper at http://www.nature.com/ naturecommunications

Competing interests: The authors declare no competing financial interests.

Reprints and permission information is available online at http://npg.nature.com/ reprintsandpermissions/

How to cite this article: Martin, M. et al. De novo evolved interference competition promotes the spread of biofilm defectors. Nat. Commun. 8, 15127 doi: $10.1038 /$ ncomms15127 (2017).

Publisher's note: Springer Nature remains neutral with regard to jurisdictional claims in published maps and institutional affiliations.

(c) (i) article are included in the article's Creative Commons license, unless indicated otherwise in the credit line; if the material is not included under the Creative Commons license, users will need to obtain permission from the license holder to reproduce the material. To view a copy of this license, visit http://creativecommons.org/licenses/by/4.0/

(C) The Author(s) 2017 